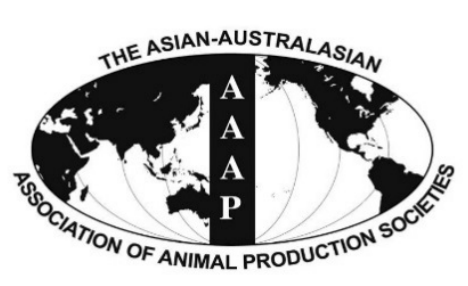

Open Access

Asian Australas. J. Anim. Sci.

Vol. 29, No. 10 : 1515-1521 October 2016

http://dx.doi.org/10.5713/ajas.15.0864

Www.ajas.info

pISSN 1011-2367 elSSN 1976-5517

\title{
Identification of Gene Expression Signatures in the Chicken Intestinal Intraepithelial Lymphocytes in Response to Herb Additive Supplementations
}

\author{
Kyeong-Hye Won ${ }^{\text {a }}$ Ki-Duk Songa ${ }^{\text {a }}$, Jong-Eun Park ${ }^{1}$, Duk-Kyung Kim ${ }^{2}$, and Chong-Sam Na* \\ Department of Animal Biotechnology, ChonBuk National University, Jeonju 54896, Korea
}

\begin{abstract}
Anethole and garlic have an immune modulatory effects on avian coccidiosis, and these effects are correlated with gene expression changes in intestinal epithelial lymphocytes (IELs). In this study, we integrated gene expression datasets from two independent experiments and investigated gene expression profile changes by anethole and garlic respectively, and identified gene expression signatures, which are common targets of these herbs as they might be used for the evaluation of the effect of plant herbs on immunity toward avian coccidiosis. We identified 4,382 and 371 genes, which were differentially expressed in IELs of chickens supplemented with garlic and anethole respectively. The gene ontology (GO) term of differentially expressed genes (DEGs) from garlic treatment resulted in the biological processes (BPs) related to proteolysis, e.g., "modification-dependent protein catabolic process", "proteolysis involved in cellular protein catabolic process", "cellular protein catabolic process", "protein catabolic process", and "ubiquitin-dependent protein catabolic process". In GO analysis, one BP term, "Proteolysis", was obtained. Among DEGs, 300 genes were differentially regulated in response to both garlic and anethole, and 234 and 59 genes were either up- or down-regulated in supplementation with both herbs. Pathway analysis resulted in enrichment of the pathways related to digestion such as "Starch and sucrose metabolism" and "Insulin signaling pathway". Taken together, the results obtained in the present study could contribute to the effective development of evaluation system of plant herbs based on molecular signatures related with their immunological functions in chicken IELs. (Key Words: Chicken, Coccidia, Differentially Expressed Genes, Integrative Analysis, Plant Herbs)
\end{abstract}

\section{INTRODUCTION}

Coccidia of the genus Eimeria infects the intestinal epithelia of chickens, turkeys, and some mammalian hosts. And the infection causes substantial economic losses to the poultry industry such as decreased nutrient absorption, retarded growth of broilers, and decreased egg production of layers (Lillehoj and Li, 2004; Lillehoj et al., 2007). In-feed anticoccidial drugs and antibiotics growth promoters showed effectiveness in loss of weight gain by avian coccidiosis. However, there is an increasing governmental restriction in

\footnotetext{
* Corresponding Author: Chong-Sam Na. Tel: +82-63-270-2607, Fax: +82-31-270-2614, E-mail: csna@jbnu.ac.kr

1 National Institute of Animal Science, Rural Development Administration, Jeonju 54896, Korea.

${ }^{2}$ C\&K Genomics, Seoul 08826, Korea.

a These authors contributed equally to this work.

Submitted Oct. 20, 2015; Revised Nov. 24, 2015; Accepted Dec. 18, 2015
}

the use of antibiotics in livestock production worldwide (Lillehoj and Lillehoj, 2000; Lillehoj et al., 2011). Recently, the alternative to antibiotics has been explored to enhance innate immunity of poultry (Lee et al., 2010; Lillehoj and Lee, 2012). Especially, plant extracts are considered as feed additives to reduce the risk of coccidiosis owing to the beneficial effects of phytonutrients on infectious diseases and cancer (Duke et al., 2003; Veldhuizen et al., 2006; Burt et al., 2007; Chang et al., 2008; Tsubura et al., 2011).

Garlic (Allium sativum) is a medicinal plant that has been used for the treatment and prevention of some diseases and has been shown to have antimicrobial, antitumor, antioxidant, and immunostimulatory properties (Imai et al., 1994; Tsao and Yin, 2001). The pharmacologic effects of garlic on cardiovascular diseases are mediated through alteration of cholesterol and triglyceride levels in the bloodstream (Kyo et al., 2001). The medicinal effects of garlic are derived from its flavonoid and organo-sulfur components. Studies in

Copyright @ 2016 by Asian-Australasian Journal of Animal Sciences This is an open-access article distributed under the terms of the Creative Commons Attribution Non-Commercial License (http://creativecommons.org/licenses/by-nc/3.0/), which permits unrestricted non-commercial use, distribution, and reproduction in any medium, provided the original work is properly cited. 
mammalian systems have indicated that these active pharmacologic components act on multiple intracellular signaling pathways. In chickens, garlic compounds have also been shown to lower cholesterol and exert immunomodulating and antimicrobial activities (Chowdhury et al., 2002; Kim and Kim, 2007; Choi et al., 2010; Hanieh et al., 2010). However, compared with human studies, there is relatively limited information on the use of garlic in poultry veterinary medicine.

In addition, garlic has been suggested for the treatment of parasites and other intestinal diseases (Iciek et al., 2009). For example, crude extracts of garlic reduced or eliminated Hymenolepis, Aspiculuris, Histomonas, and Eimeria parasites in animal models of infection (Hafez and Hauck, 2006; Toulah and Al-Rawi, 2007; Ayaz et al., 2008; AbdelGhaffar et al., 2011; Dkhil et al., 2011). Of these parasites, coccidia protozoa comprise a subclass of single-celled eukaryotic microorganisms belonging to the phylum Apicomplexa and class Conoidasida. Coccidia of the genus Eimeria infect the intestinal epithelia of chickens, turkeys, and some mammalian hosts. Garlic has been shown to exert anticoccidial effects in mouse and rabbit infection models (Dkhil et al., 2011).

Anethole, 1-methoxy-4-(1-propenyl) benzene, is an aromatic, unsaturated ether that occurs as a major component of the essential oils of anise (Pimpinella anisum), star anise (Illicium verum), fennel (Foeniculum vulgare), and liquorice (Glycyrrhiza glabra), and is widely used as a flavoring agent (Windholz et al., 1983). In mammalian systems, anethole had demonstrable anti-carcinogenic, anti-oxidant, and antiinflammatory activities (Al-Harbi et al., 1995; Chainy et al., 2000; Freire et al., 2005), as well as antimicrobial properties against bacteria, fungi, and nematodes (Kubo and Fujita, 2001; Lee et al., 2002). Broiler chickens fed with an anethole-supplemented diet showed resistance to coccidiosis (Kim et al., 2013a,b). These broad-spectrum pharmacologic activities suggest that anethole may also protect avian hosts against infectious diseases. However, only limited studies have been reported describing the use of anethole in veterinary medicine (Cardozo et al., 2005).

In this study, by integrating the public microarray data of the studies that investigated the effect of garlic and anethole on transcriptomes of the intestinal intraepithelial lymphocytes (IEL) of chicken, we aimed to identify the common signatures, which are targeted by both garlic and anethole to provide comprehensive strategy to evaluate plant herbs.

\section{MATERIALS AND METHODS}

\section{Microarray data acquisition}

To investigate common effects of two herbs, garlic and anethole, we used the Agilent Chicken Gene Expression Microarray dataset from two previous studies on their effects as the feed additives that were obtained from Gene Expression Omnibus. In one of the studies, a compound including garlic-derived secondary metabolites, propyl thiosulphinate and propyl thiosulphinate oxide, was added to the feed to enhance intestinal immunity of chicken (accession number GSE36302) (Kim et al., 2013a). The other study used anethole as the dietary supplement (accession number GSE41250) (Kim et al., 2013b). The design of microarray image analysis of both studies was a comparison of treatment (garlic and anethole) with non-supplemented control, identifying immunological effects of herbs.

The dataset used in this study was in reference to these previous studies, which contains details on feeding procedures of each herb (Kim et al., 2013a, b). The feeding procedures of each herb have been reported in Kim et al. studies (2013a, b). Briefly, chickens were fed from hatch with a diet supplemented with each herb or a standard diet alone (control). At 14 days post hatch, IEL were isolated from chickens given the herb-supplemented or nonsupplemented diets. Total RNAs were isolated from the cells using Trizol (Invitrogen, Carlsbad, CA, USA) and amplified with cyanine 3 (Cy3)- or Cy5-labeled CTP. Then, the labeled RNAs were hybridized to a Chicken Gene Expession Microarray (Agilent Technologies, Santa Clara, CA, USA). In this study, microarray image analysis was performed to identify common and specific effects of two different herbs on transcriptional changes in chicken gut.

\section{Identification and functional analysis of differentially expressed genes}

$\mathrm{R}$ package 'limma' was used to normalize and qualify microarray images. Median signal intensities were corrected by adaptive background correction (Ritchie et al., 2007) and normalized by locally-weighted scatterplot smoothing method. The log2-transformed fold changes and standard errors were estimated by fitting a linear model and empirical Bayes statistics was applied for smoothing standard errors. Differentially expressed genes (DEG) were filtered by cutoff 0.1 of false discovery rate, with adjusted p-value of twosample t-test. Annotation of DEGs and biological function analysis were performed using DAVID Bioinformatics Resources (http://david.abcc.ncifcrf.gov/).

\section{RESULTS}

\section{Identification of differentially expressed genes}

In the present study, microarray datasets from two independent studies were combined to analyze the discriminative effects of two different herbs, garlic, and anethole. Both of herbs were fed to the chickens as feed 
additives. In the results, garlic-fed chickens had greater number of modified genes than those fed with anethole. The numbers of DEGs were 4,682 (2,613 genes upregulated and 2,069 genes downregulated) and 371 (271 genes upregulated and 100 genes downregulated) in the supplementation of garlic-derived compound and anethole, respectively. The heatmap and hierarchical clustering for each experiment was displayed in supplemental Figure 1. The number of common DEGs found by both garlic and anethole additives was 300 (Figure 1). Detailed information for common DEGs was described in supplemental Table 1. The DEGs were used to identify their biological functions and the pathways in which the genes are involved.

\section{Biological function analysis}

In the gene ontology (GO) analysis, 38 and 1 terms of biological process (BPs) were significantly identified from the DEGs by the treatment of garlic and anethole, respectively (Table 1). The only BP that was classified from the DEGs by anethole treatment was the term of "Proteolysis". Among the BPs from the DEGs by garlic treatment, there were several terms related with proteolysis such as "Modification-dependent protein catabolic process", "Proteolysis involved in cellular protein catabolic process", "Cellular protein catabolic process", "Protein catabolic process", and "Ubiquitin-dependent protein catabolic process".

(A)

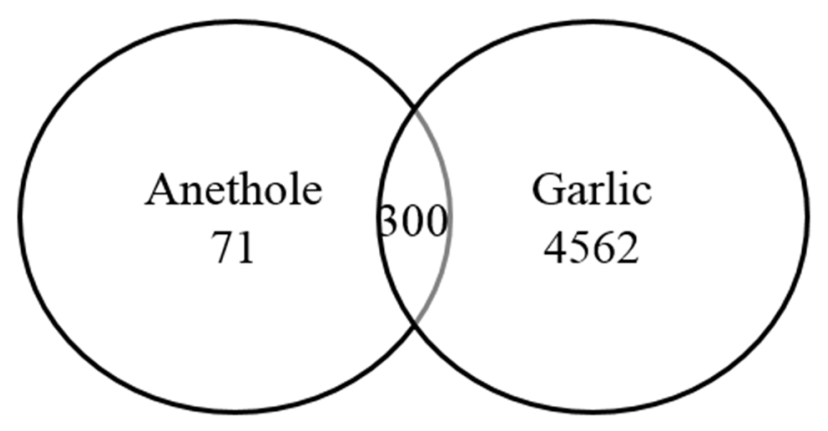

(B)

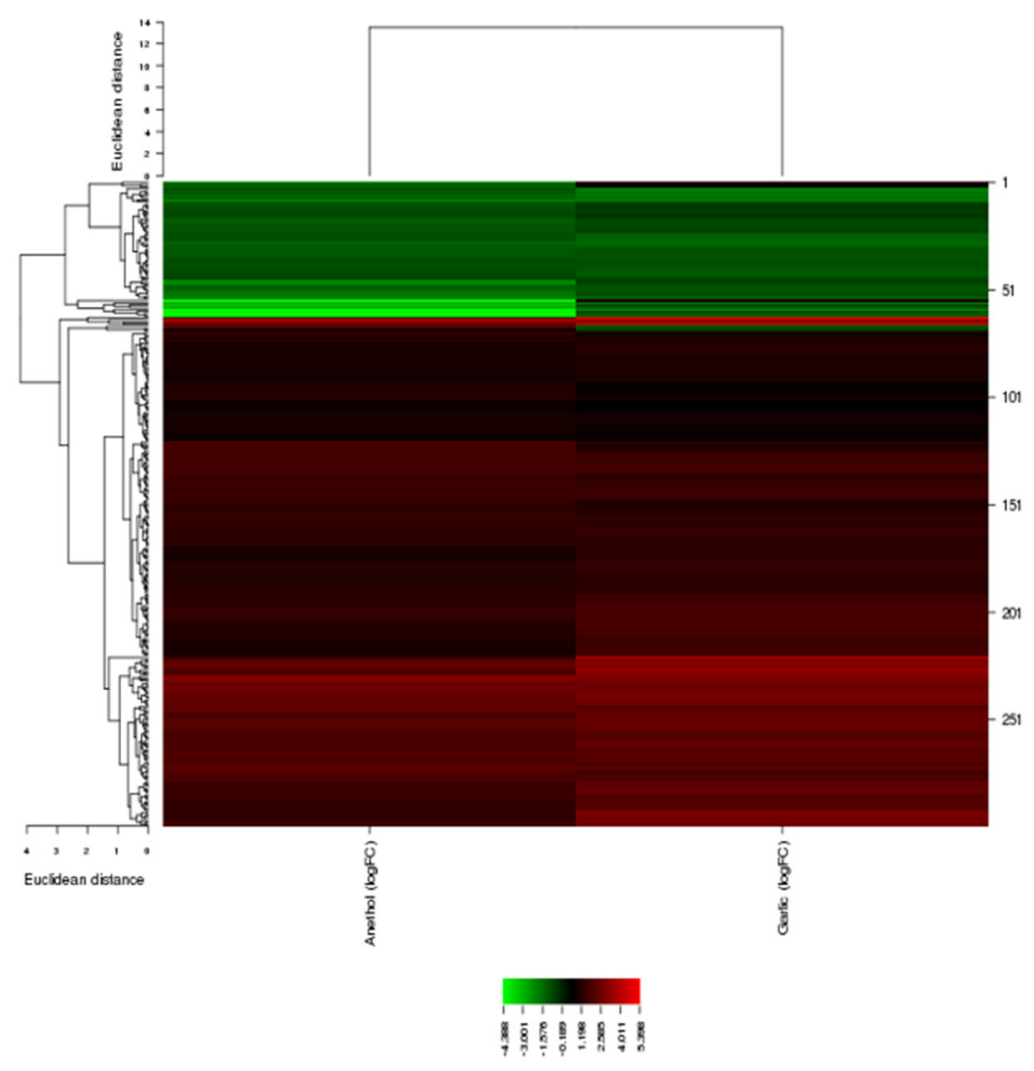

Figure 1. Common target genes that were differentially expressed in chicken intestinal intraepithelial lymphocytes (IELs) by anethole and garlic supplementations. (A) Venn diagram showing the shared differentially expressed genes (DEGs) by the treatment of two different herbs (false discovery rate $[F D R]<0.1$ ). (B) Heat-map of common target gene expression, which were differentially expressed from chicken IELs in response to herbs. 
Table 1. Significant biological processes (BPs) for the differentially expressed genes (DEGs) in the treatment of anethole and garlic using DAVID

\begin{tabular}{|c|c|c|c|}
\hline Herbs & Biological process & p-value & No. of genes \\
\hline Anethole & Proteolysis & $3.09 \mathrm{E}-02$ & 10 \\
\hline \multirow[t]{38}{*}{ Garlic } & Modification-dependent macromolecule catabolic process & $1.03 \mathrm{E}-04$ & 28 \\
\hline & Modification-dependent protein catabolic process & $1.03 \mathrm{E}-04$ & 28 \\
\hline & Proteolysis involved in cellular protein catabolic process & $6.95 \mathrm{E}-04$ & 28 \\
\hline & Cellular protein catabolic process & $8.85 \mathrm{E}-04$ & 28 \\
\hline & Protein catabolic process & $1.57 \mathrm{E}-03$ & 29 \\
\hline & Blastocyst development & $2.53 \mathrm{E}-03$ & 9 \\
\hline & Macromolecule catabolic process & 2.61E-03 & 34 \\
\hline & Cellular macromolecule catabolic process & 2.81E-03 & 31 \\
\hline & Ubiquitin-dependent protein catabolic process & $3.75 \mathrm{E}-03$ & 17 \\
\hline & Transcription & $5.54 \mathrm{E}-03$ & 55 \\
\hline & Embryonic epithelial tube formation & $6.42 \mathrm{E}-03$ & 7 \\
\hline & Tube lumen formation & $6.42 \mathrm{E}-03$ & 7 \\
\hline & Lipid biosynthetic process & $8.56 \mathrm{E}-03$ & 18 \\
\hline & Positive regulation of cell adhesion & $8.78 \mathrm{E}-03$ & 7 \\
\hline & Regulation of programmed cell death & $1.04 \mathrm{E}-02$ & 35 \\
\hline & Regulation of cell death & $1.11 \mathrm{E}-02$ & 35 \\
\hline & Cellular response to extracellular stimulus & $1.21 \mathrm{E}-02$ & 6 \\
\hline & Regulation of apoptosis & $1.34 \mathrm{E}-02$ & 34 \\
\hline & Amine biosynthetic process & $1.56 \mathrm{E}-02$ & 11 \\
\hline & Neural tube formation & $1.66 \mathrm{E}-02$ & 6 \\
\hline & Phospholipid biosynthetic process & $1.94 \mathrm{E}-02$ & 7 \\
\hline & Cofactor biosynthetic process & $2.11 \mathrm{E}-02$ & 11 \\
\hline & Blastocyst formation & $2.31 \mathrm{E}-02$ & 5 \\
\hline & Trophectodermal cell differentiation & $2.31 \mathrm{E}-02$ & 5 \\
\hline & Response to hypoxia & 2.44E-02 & 7 \\
\hline & Morphogenesis of an epithelium & 2.79E-02 & 11 \\
\hline & Tube development & 2.84E-02 & 19 \\
\hline & Response to oxygen levels & $3.01 \mathrm{E}-02$ & 7 \\
\hline & Positive regulation of cell-cell adhesion & $3.18 \mathrm{E}-02$ & 3 \\
\hline & Embryonic process involved in female pregnancy & $3.18 \mathrm{E}-02$ & 3 \\
\hline & Embryonic placenta development & $3.18 \mathrm{E}-02$ & 5 \\
\hline & Epithelial tube morphogenesis & $3.53 \mathrm{E}-02$ & 8 \\
\hline & Cellular amino acid biosynthetic process & $3.66 \mathrm{E}-02$ & 7 \\
\hline & Mammary gland development & $3.66 \mathrm{E}-02$ & 7 \\
\hline & Glutamine family amino acid metabolic process & $3.66 \mathrm{E}-02$ & 7 \\
\hline & Respiratory system development & 4.09E-02 & 11 \\
\hline & Primary neural tube formation & 4.22E-02 & 5 \\
\hline & Morphogenesis of embryonic epithelium & $4.40 \mathrm{E}-02$ & 7 \\
\hline
\end{tabular}

Figure 2 showed the correlated expression of the common DEGs changed by feeding of both herbs. Among the 300 common DEGs, 234 and 59 genes were up- and down-regulated in the treatment of both herbs, respectively. The remaining 7 genes did not show any correlated expression for both herbs. The terms of BPs, cellular components and molecular functions (MFs) and by GO analysis for the DEGs with same directional expression changes by garlic and anethole were listed in Table 2, 3, and 4 , respectively. KEGG pathway terms enriched by common DEGs from the treatment of two different herbs were listed in Table 5. Both herbs showed common effects on 10 pathways, including adipocytokine signaling, vascular smooth muscle contraction, focal adhesion, starch and sucrose metabolism, drug metabolism, metabolism of xenobiotics by cytochrome P450, tight junction, insulin signaling pathway, glutathione metabolism and lysine degradation (Table 5).

\section{DISCUSSIONS}

For the comparative analysis of the effects of two 


\section{Correlated expression between Anethol and Garlic}

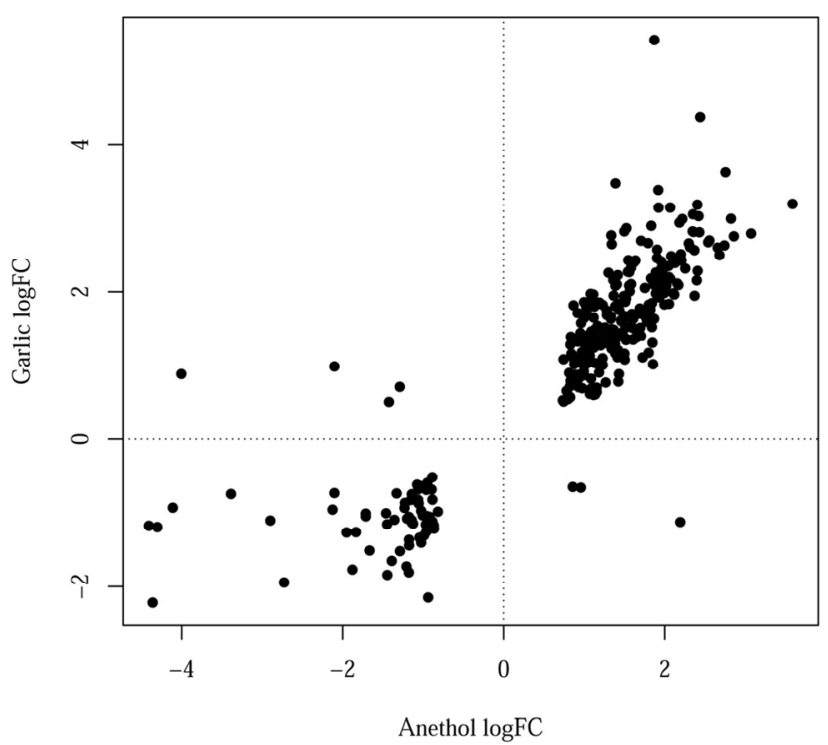

Figure 2. Correlated expression of the differentially expressed genes (DEGs) between anethole and garlic.

different herbs, we collected microarray datasets from the independent trials for each herb, garlic and anethole, and identified GO terms and pathways enriched by the DEGs. In terms of number of DEGs, garlic showed more gene expression than anethole. In the present study, the most enriched category of BP affected by anethole was "Proteolysis" and those of BPs by garlic contained 8 processes related with "Catabolic processes" (Table 1). Proteolysis is the breakdown of proteins into smaller polypeptides or amino acids. The catabolic process refers to the chemical reactions and pathways resulting in the breakdown of substances, including the breakdown of carbon compounds with the liberation of energy for use by the cell or organism (De Bolster, 1997). These two GO terms are related to feed intake. The improvement of feed intake and daily live weight gain was observed in $1 \%$ anise supplement in Broiler chicken (Al-Kassie, 2008). This improvement may be due to the appetizing effect of active ingredient (such as

Table 2. Significant biological processes (BPs) for differentially expressed genes (DEGs) in the treatment of anethole and garlic using DAVID

\begin{tabular}{lcc}
\hline Biological process & Count & p-value \\
\hline Up-regulated & & \\
$\quad$ Regulation of transcription & 12 & $1.80 \mathrm{E}-02$ \\
Transcription & 7 & $2.80 \mathrm{E}-02$ \\
Regulation of cell migration & 3 & $4.70 \mathrm{E}-02$ \\
Regulation of transcription, & 9 & $4.70 \mathrm{E}-02$ \\
$\quad$ DNA-dependent & & \\
Down-regulated & & \\
$\quad$ Proteolysis & 5 & $3.00 \mathrm{E}-03$ \\
$\quad$ Digestion & 2 & $2.40 \mathrm{E}-02$ \\
\hline
\end{tabular}

Table 3. Significant cellular components (CCs) for differentially expressed genes (DEGs) in the treatment of anethole and garlic using DAVID

\begin{tabular}{lcc}
\hline Cellular component & Count & p-value \\
\hline Up-regulated & & \\
$\quad$ extracellular region & 7 & $9.20 \mathrm{E}-01$ \\
cell fraction & 3 & $9.9 \mathrm{E}-01$ \\
microsome & 2 & $9.8 \mathrm{E}-01$ \\
vesicular fraction & 2 & $9.5 \mathrm{E}-01$ \\
extracellular space & 3 & $9.2 \mathrm{E}-01$ \\
$\quad$ extracellular region part & 4 & $8.8 \mathrm{E}-01$ \\
Down-regulated & & \\
$\quad$ extracellular space & 3 & $4.70 \mathrm{E}-01$ \\
$\quad$ extracellular region & 4 & $4.90 \mathrm{E}-01$ \\
$\quad$ extracellular region part & 3 & $5.20 \mathrm{E}-01$ \\
\hline
\end{tabular}

anethole) in anise (Cabuk et al., 2003). In previous studies, garlic powder supplement and oil improved feed digestibility in cattle and sheep (Klevenhusen et al., 2011; Manasri et al., 2012). Therefore, the results suggest that anethole and garlic may function as important herbs for feed digestibility in chicken.

Interestingly, most of common DEGs by the treatment of anethole and garlic showed same directional changes in gene expression (Figure 2). Furthermore, in GO analysis in Table 2,3 , and 4 , for the DEGs with same direction changes, the BPs identified from the down-regulated genes by anethole and garlic were "Proteolysis" and "Digestion". The MFs from the down-regulated genes by both herbs were "Glutathione transferase activity", "Peptidase activity, acting on L-amino acid peptides" and "Peptidase activity". Together, the two herbs analyzed in this study could have important roles in the function of digestion in the chicken gut and it may be regulated by the down-regulated genes.

In the pathway analysis (Table 5), the DEGs, which were altered by both herbs constitute the pathways related to digestion such as "Starch and sucrose metabolism" and "Insulin signaling pathway". "Glutathione metabolism" is critical for the development of effective strategies to improve

Table 4. Significant molecular functions for differentially expressed genes (DEGs) in the treatment of anethole and garlic using DAVID

\begin{tabular}{lcc}
\hline Molecular function & Count & p-value \\
\hline Up/up-regulated & & \\
DNA binding & 12 & $2.10 \mathrm{E}-02$ \\
Transcription regulator activity & 10 & $2.30 \mathrm{E}-02$ \\
$\quad$ Transcription factor activity & 8 & $2.80 \mathrm{E}-02$ \\
Down/down-regulated & & \\
$\quad$ Glutathione transferase activity & 2 & $1.90 \mathrm{E}-02$ \\
$\quad$ Peptidase activity, acting on & 4 & $2.90 \mathrm{E}-02$ \\
$\quad$ L-amino acid peptides & & \\
$\quad$ Peptidase activity & 4 & $3.20 \mathrm{E}-02$ \\
\hline
\end{tabular}


Table 5. Pathway analysis for the differentially expressed genes (DEGs) in the treatment of two types of herbs simultaneously

\begin{tabular}{ll}
\hline KEGG pathway & Genes \\
\hline Adipocytokine signaling pathway & IRS-1, SOCS3, ADIPOR2 \\
Vascular smooth muscle contraction & RCJMB04_22k13, LOC421179, LOC419752 \\
Focal adhesion & GRB2, RCJMB04_22k13, LOC417506, LOC395210 \\
Starch and sucrose metabolism & AMY1C,UGP2 \\
Drug metabolism & GSTT1, GSTA1 \\
Metabolism of xenobiotics by cytochrome P450 & GSTT1, GSTA1 \\
Tight junction & LOC417506, LOC418790, RRAS2 \\
Insulin signaling pathway & IRS-1, GRB2, SOCS3 \\
Glutathione metabolism & GSTT1, GSTA1 \\
Lysine degradation & WHSC1L1, LOC416214 \\
\hline
\end{tabular}

health and many diseases since glutathione plays important roles in antioxidant defense, nutrient metabolism, and regulation of cellular events (including gene expression, DNA and protein synthesis, cell proliferation and apoptosis, signal transduction, cytokine production and immune response, and protein glutathionylation) (Wu et al., 2004). Glutathione deficiency contributes to oxidative stress, which plays a key role in aging and the pathogenesis of many diseases (including kwashiorkor, seizure, Alzheimer's disease, Parkinson's disease, liver disease, cystic fibrosis, sickle cell anemia, HIV/AIDS, cancer, heart attack, stroke, and diabetes) (Townsend et al., 2003). Therefore, the common digestive properties of anethole and garlic may be utilized as feed supplement for chicken to improve the animal's health.

\section{CONFLICT OF INTEREST}

We certify that there is no conflict of interest with any financial organization regarding the material discussed in the manuscript.

\section{ACKNOWLEDGMENTS}

This work was supported by a grant from the NextGeneration BioGreen 21 Program (No. PJ01104401), Rural Development Administration, Republic of Korea.

\section{REFERENCES}

Abdel-Ghaffar, F., M. Semmler, K. A. S. Al-Rasheid, B. Strassen, K. Fischer, G. Aksu, S. Klimpel, and H. Mehlhorn. 2011. The effects of different plant extracts on intestinal cestodes and on trematodes. Parasitol. Res. 108:979-984.

al-Harbi, M. M., S. Qureshi, M. Raza, M. M. Ahmed, A. B. Giangreco, and A. H. Shah. 1995. Influence of anethole treatment on the tumour induced by Ehrlich ascites carcinoma cells in paw of Swiss albino mice. Eur. J. Cancer Prev. 4:307318.

Al-Kassie, G. A. M. 2008. The effect of anise and rosemary on broiler performance. Int. J. Poult. Sci. 7:243-245.

Ayaz, E., I. Turel, A. Gul, and O. Yilmaz, 2008. Evaluation of the anthelmentic activity of garlic (Allium sativum) in mice naturally infected with Aspiculuris tetraptera. Recent Pat. Antiinfect. Drug Discov. 3:149-152.

Burt, S. A., M. J. Fledderman, H. P. Haagsman, F. van Knapen, and E. J. A. Veldhuizen. 2007. Inhibition of Salmonella enterica serotype Enteritidis on agar and raw chicken by carvacrol vapour. Int. J. Food Microbiol. 119:346-350.

Cabuk, M., A. Alcicek, M. Bozkurt, and N. Imre. 2003. Antimicrobial properties of the essential oils isolated from aromatic plants and using possibility as alternative feed additives. In: Paper presented at the II. National Animal Nutrition Congress, the National Animal Nutrition Congress, Konya, Turkey. pp. 18-20.

Cardozo, P. W., S. Calsamiglia, A. Ferret, and C. Kamel. 2005. Screening for the effects of natural plant extracts at different $\mathrm{pH}$ on in vitro rumen microbial fermentation of a high-concentrate diet for beef cattle. J. Anim. Sci. 83:2572-2579.

Chainy, G. B., S. K. Manna, M. M. Chaturvedi, and B. B. Aggarwal. 2000. Anethole blocks both early and late cellular responses transduced by tumor necrosis factor: effect on NF-kappaB, AP1, JNK, MAPKK and apoptosis. Oncogene 19:2943-2950.

Chang, C. W., W. L. Chang, S. T. Chang, and S. S. Cheng. 2008. Antibacterial activities of plant essential oils against Legionella pneumophila. Water Res. 42:78-286.

Choi, I. H., W. Y. Park, and Y. J. Kim. 2010. Effects of dietary garlic powder and \{alpha\}-tocopherol supplementation on performance, serum cholesterol levels, and meat quality of chicken. Poult. Sci. 89:1724-1731.

Chowdhury, S. R., S. D. Chowdhury, and T. K. Smith. 2002. Effects of dietary garlic on cholesterol metabolism in laying hens. Poult. Sci. 81:1856-1862.

De Bolster, M. 1997. Glossary of terms used in bioinorganic chemistry (IUPAC recommendations 1997). Pure Appl. Chem. 69:1251-1303.

Dkhil, M. A., A. S. Abdel-Baki, F. Wunderlich, H. Sies, and S. AlQuraishy. 2011. Anticoccidial and antiinflammatory activity of garlic in murine Eimeria papillata infections. Vet. Parasitol. 175:66-72.

Duke, S. O., S. R. Baerson, F. E. Dayan, A. M. Rimando, B. E Scheffler, M. R. Tellez, D. E. Wedge, K. K. Schrader, D. H. Akey, and F. H. Arthur, et al. 2003. United States Department of Agriculture-Agricultural Research Service research on natural 
products for pest management. Pest. Manag. Sci. 59:708-717.

Freire, R. S., S. M. Morais, F. E. Catunda-Junior, and D. C. Pinheiro. 2005. Synthesis and antioxidant, anti-inflammatory and gastroprotector activities of anethole and related compounds. Bioorg. Med. Chem. 13:4353-4358.

Hafez, H. M. and R. Hauck. 2006. Efficacy of a herbal product against Histomonas meleagridis after experimental infection of turkey poults. Arch. Anim. Nutr. 60:436-442.

Hanieh, H., K. Narabara, M. Piao, C. Gerile, A. Abe, and Y. Kondo. 2010. Modulatory effects of two levels of dietary Alliums on immune response and certain immunological variables, following immunization, in White Leghorn chickens. Anim. Sci. J. 81:673-680.

Iciek, M., I. Kwiecien, and L. Wlodek. 2009. Biological properties of garlic and garlic-derived organosulfur compounds. Environ. Mol. Mutagen. 50:247-265.

Imai, J., N. Ide, S. Nagae, T. Moriguchi, H. Matsuura, and Y. Itakura. 1994. Antioxidant and radical scavenging effects of aged garlic extract and its constituents. Planta Med. 60:417-420.

Kim, D. K., H. S. Lillehoj, S. H. Lee, S. I. Jang, M. S. Park, W. Min, E. P. Lillehoj, and D. Bravo. 2013a. Immune effects of dietary anethole on Eimeria acervulina infection. Poult. Sci. 92:26252634.

Kim, D. K., H. S. Lillehoj, S. H. Lee, E. P. Lillehoj, and D. Bravo. 2013b. Improved resistance to Eimeria acervulina infection in chickens due to dietary supplementation with garlic metabolites. Br. J. Nutr. 109:76-88.

Kim, J. S. and Y. Kim. 2007. The inhibitory effect of natural bioactives on the growth of pathogenic bacteria. Nutr. Res. Pract. 1:273-278.

Klevenhusen, F., J. O. Zeitz, S. Duval, M. Kreuzer, and C. R. Soliva. 2011. Garlic oil and its principal component diallyl disulfide fail to mitigate methane, but improve digestibility in sheep. Anim. Feed Sci. Technol. 166:356-363.

Kubo, I. and K. Fujita. 2001. Naturally occurring anti-Salmonella agents. J. Agric. Food Chem. 49:5750-5754.

Kyo, E., N. Uda, S. Kasuga, and Y. Itakura. 2001. Immunomodulatory effects of aged garlic extract. J. Nutr. 131:1075S-1079S.

Lee, P. D., R. Sladek, C. M. Greenwood, and T. J. Hudson. 2002. Control genes and variability: Absence of ubiquitous reference transcripts in diverse mammalian expression studies. Genome Res. 12:292-297.

Lee, S. H., H. S. Lillehoj, S. I. Jang, D. K. Kim, C. Ionescu, and D. Bravo. 2010. Effect of dietary Curcuma, Capsicum, and Lentinus on enhancing local immunity against Eimeria acervulina infection. J. Poult. Sci. 47:89-95.

Lillehoj, H. S., C. H. Kim, C. L. Keeler Jr., and S. Zhang. 2007. Immunogenomic approaches to study host immunity to enteric pathogens. Poult. Sci. 86:1491-1500.
Lillehoj, H. S., D. K. Kim, D. M. Bravo, and S. H. Lee. 2011. Effects of dietary plant-derived phytonutrients on the genome-wide profiles and coccidiosis resistance in the broiler chickens. BMC Proc. 5:S34.

Lillehoj, H. S. and K. W. Lee. 2012. Immune modulation of innate immunity as alternatives-to-antibiotics strategies to mitigate the use of drugs in poultry production. Poult. Sci. 91:1286-1291.

Lillehoj, H. S. and G. Li. 2004. Nitric oxide production by macrophages stimulated with Coccidia sporozoites, lipopolysaccharide, or interferon-gamma, and its dynamic changes in SC and TK strains of chickens infected with Eimeria tenella. Avian Dis. 48:244-253.

Lillehoj, H. S. and E. P. Lillehoj. 2000. Avian coccidiosis. A review of acquired intestinal immunity and vaccination strategies. Avian Dis. 44:408-425.

Manasri, N., M. Wanapat, and C. Navanukraw. 2012. Improving rumen fermentation and feed digestibility in cattle by mangosteen peel and garlic pellet supplementation. Livest. Sci. 148:291-295

O'Gara, E. A., D. J. Hill, and D. J. Maslin. 2000. Activities of garlic oil, garlic powder, and their diallyl constituents against Helicobacter pylori. Appl. Environ. Microbiol. 66:2269-2273.

Ritchie, M. E., J. Silver, A. Oshlack, M. Holmes, D. Diyagama, A. Holloway, and G. K. Smyth. 2007. A comparison of background correction methods for two-colour microarrays. Bioinformatics 23:2700-2707.

Rivlin, R. S. 2001. Historical perspective on the use of garlic. J. Nutr. 131:951S-954S.

Toulah, F. H. and M. M. Al-Rawi. 2007. Efficacy of garlic extract on hepatic coccidiosis in infected rabbits (Oryctolagus cuniculus): histological and biochemical studies. J. Egypt Soc. Parasitol. 37:957-968.

Townsend, D. M., K. D. Tew, and H. Tapiero. 2003. The importance of glutathione in human disease. Biomed. Pharmacother. 57:145-155.

Tsao, S. M. and M. C. Yin. 2001. In-vitro antimicrobial activity of four diallyl sulphides occurring naturally in garlic and Chinese leek oils. J. Med. Microbiol. 50:646-649.

Tsubura, A., Y. C. Lai, M. Kuwata, N. Uehara, and K. Yoshizawa. 2011. Anticancer effects of garlic and garlic-derived compounds for breast cancer control. Anticancer Agents Med. Chem.11:249-253.

Veldhuizen, E. J. A., J. L. M. Tjeerdsma-van Bokhoven, C. Zweijtzer, S. A. Burt, and H. P. Haagsman. 2006. Structural requirements for the antimicrobial activity of carvacrol. J. Agric. Food. Chem. 54:1874-1879.

Windholz, M., S. Budavari, R. F. Blumetti, and E. S. Otterbein. 1983. The Merck Index. 10th edn. Merck and Co. Inc., Rahway, NJ, USA.

Wu, G., Y. Z. Fang, S. Yang, J. R. Lupton, and N. D. Turner. 2004. Glutathione metabolism and its implications for health. J. Nutr. 134:489-492. 